\title{
The rate of co-infection for piglet diarrhea viruses in China and the genetic characterization of porcine epidemic diarrhea virus and porcine kobuvirus
}

\author{
Z.-P. ZHAO ${ }^{1 *}$, Z. YANG ${ }^{1 *}$, W.-D. LIN ${ }^{1}$, W.-Y. WANG ${ }^{1}$, J. YANG ${ }^{1}$, W.-J. JIN ${ }^{1,2^{*}}$, A.-J. QIN ${ }^{1 *}$
}

\begin{abstract}
${ }^{1}$ Ministry of Education Key Lab for Avian Preventive Medicine, Jiangsu Co-innovation Center for Prevention and Control of Important Animal Infectious Diseases and Zoonoses, College of Veterinary Medicine, Yangzhou university, Jiangsu, Yangzhou 225009, P. R. China; ${ }^{2}$ College of Veterinary Medicine, China Agricultural University, Beijing, 100193, P. R. China
\end{abstract}

\begin{abstract}
Summary. - Piglet diarrhea epidemics result in major economic losses for the swine industry. Four viruses are closely linked to porcine diarrhea: porcine kobuvirus (PKV), porcine epidemic diarrhea virus (PEDV), porcine transmissible gastroenteritis virus (TGEV), and porcine rotavirus (PRoV). We have conducted an epidemiology study to determine the frequency of infection and co-infection with these viruses in China, and characterized the genetic variation of the isolated PEDV and PKV strains. Stool and intestinal samples $(\mathrm{n}=314)$ were collected from piglets with diarrhea in China from years 2012 to 2014. RT-PCR was used to detect PKV, PEDV, TGEV, and PRoV. Phylogenetic relationships between reference strains and the isolated PEDV and PKV strains were determined based on the $\mathrm{M}$ and 3D gene sequence. The rates of infection with PKV, PEDV, TGEV and PRoV were $29.9 \%, 24.2 \%, 1.91 \%$, and $0.31 \%$, respectively. Co-infections with PKV and the other three viruses were very common. Co-infection of PKV and PEDV was detected in $15.0 \%$ (47/314) of the samples. Phylogenetic analysis of the PKV 3D gene indicated that there were some phylogenetic differences in the PKV strains across regions within China. However, according to the PEDV M gene, strains clustered into three groups and the primary group was distinct from the vaccine strain CV777. This study provides insights in to the prevalence of diarrhea viruses and their prevention and control in China.
\end{abstract}

Keywords: porcine diarrhea viruses; co-infection; porcine kobuvirus; porcine epidemic diarrhea virus; porcine gastroenteritis; porcine rotavirus

\section{Introduction}

Viral diarrhea is a major cause of morbidity and mortality in piglets. The principal etiological agents of most viral diarrhea and malabsorption diseases in piglets are: transmissible gastroenteritis virus (TGEV), porcine epidemic diarrhea virus (PEDV), and porcine rotavirus (PRoV). PEDV and TGEV are enveloped, single-stranded RNA viruses belonging

"Corresponding authors: E-mail: wenjiejin1@163.com, aijian@ yzu.edu.cn; phone: +8613815809571 . " These authors contributed equally to this work.

Abbreviations: $\mathrm{PKV}=$ porcine kobuvirus; $\mathrm{PEDV}=$ porcine epidemic diarrhea virus; TGEV = porcine transmissible gastroenteritis virus; ProV = porcine rotavirus to the Coronaviridae family. PRoV is a double-stranded, nonenveloped RNA virus belonging to the Reoviridae family. All three viruses cause an acute and highly contagious enteric disease characterized by severe enteritis, watery diarrhea, vomiting, and dehydration that is associated with a high degree of mortality in suckling piglets (Coussement et al., 1982; Laude et al., 1990; Prabha and Verghese, 2009).

A fourth possible etiological agent of diarrhea in piglets is porcine kobuvirus (PKV), a member of the Kobuvirus genus in the Picornaviridae family. PKV is a non-enveloped, singlestranded, positive-sense RNA virus that was first detected in Hungary in early 2007 (Reuter et al., 2008), and has now been detected worldwide (Khamrin et al., 2010; Sisay et al., 2013; Yu et al., 2009). While the pathogenesis of PKV is relatively unknown, Park et al. suggested that PKV could be the etiological agent of gastroenteritis in pigs based on 
statistical analysis (Park et al., 2010). Infections with PEDV, PKV, TGEV, and PRoV were all reported in an epidemic of porcine diarrhea in 2010 in China (Cao et al., 2012; Shi et al., 2013; Zhao et al., 2013).

Co-infection with these viruses makes preventing and curing diarrhea in pigs more complex. Therefore, we performed an epidemiological study to identify the frequency of these viruses in cases of porcine viral diarrhea in China. We also characterized the genetic variation and molecular epidemiology of PEDV and PKV, which represent major potential threats. The membrane $(\mathrm{M})$ protein of PEDV and 3D protein of PKV were conservative but important for viral replication, and they were therefore chosen for phylogenetic analysis.

\section{Materials and Methods}

Sample collection. Intestine or stool samples $(\mathrm{n}=314)$ were collected from piglets with acute enteritis or watery diarrhea collected from throughout Jilin, Henan, Jiangsu, Shanghai, and Guangdong provinces between the years 2012 and 2014. The samples were diluted nine-times by phosphate-buffered saline (PBS, $0.1 \mathrm{M}, \mathrm{pH}$ 7.2 ), vortexed, and then centrifuged at $2,000 \times \mathrm{g}$ for $10 \mathrm{~min}$ at $4^{\circ} \mathrm{C}$. The supernatants were then stored at $-80^{\circ} \mathrm{C}$ until they were used for analysis. The experiments were performed according to the institutional animal care guidelines and approved by the Animal Care Committee of College of Veterinary Medicine, Yangzhou University.

$R T-P C R$. Total RNA was extracted from the stored sample supernatants using TRIzol (Invitrogen, USA) according to the manufacturer's instructions. Reverse transcription was performed using the SuperScript III first-strand synthesis system (Invitrogen, USA), and then the synthesized CDNA was used for PCR. Primer sequences were designed to detect PKV, PEDV, TGEV, and PRoV based on the reference sequence for each virus (Table1). PCR reactions were carried out in a $25 \mu$ l volume in PCR buffer containing LA Taq polymerase (2.5 U; TaKaRa, China), $\mathrm{MgCl}_{2}(1.5 \mathrm{mmol} / \mathrm{l})$, template DNA (20 ng), the forward and reverse primers $(10 \mu \mathrm{mol} / \mathrm{l}$ each), and dNTPs ( $2.5 \mu \mathrm{mol} / \mathrm{l}$ each). The fragments were amplified using the following reaction conditions: one cycle at $95^{\circ} \mathrm{C}$ for 5 min; followed by 35 cycles consisting of denaturation at $95^{\circ} \mathrm{C}$ for $1 \mathrm{~min}$, annealing at $55^{\circ} \mathrm{C}(\mathrm{PEDV}, \mathrm{TGEV}), 52^{\circ} \mathrm{C}(\mathrm{PKV})$, or $53.5^{\circ} \mathrm{C}$ (PRoV) for $45 \mathrm{sec}$, and amplification at $72^{\circ} \mathrm{C}$ for $1 \mathrm{~min}$; and a final extension step lasting $10 \mathrm{~min}$ at $72^{\circ} \mathrm{C}$. PCR products were then visualized on a $1.2 \%$ agarose gel.

DNA cloning and sequence analysis. To investigate the molecular epidemiology and genetic variation of PEDV and PKV, the bands from 16 PEDV-positive samples and 15 PKV-positive samples (Table 2) were cloned and sequenced. The bands were excised from the $1.2 \%$ agarose gel, and then purified using the QIAquick gel extraction kit (QIAGEN, Germany) according to the manufacturer's instructions. The purified DNA was cloned into the $\mathrm{PGEM}^{\circ}-\mathrm{T}$ Easy vector (Promega, USA), and these constructs were used to transform E. coli DH5a competent cells. The resulting plasmids were sequenced by Invitrogen. Finally, the nucleotide sequences and deduced amino acid sequences were compared to other PEDV and kobuviruses in GenBank (Table 3). Sequence similarity analysis was performed using the ClustalW method using the Megalign 7.2 program. Phylogenetic analysis was carried out based on nucleotide alignments using the MEGA 5.0 software (Tamura et al., 2011).

\section{Results}

Virus detection in samples collected from the field

Of the 314 samples collected in the field 94 (29.9\%) were positive for PKV, 76 (24.2\%) were positive for PEDV, $6(1.91 \%)$ were positive for TGEV, and only $1(0.31 \%)$ was positive for PRoV. Infection with PKV alone was detected in $45 / 314$ samples $(14.3 \%)$. The remaining PKV positive samples $(49 / 94 ; 52.1 \%)$ were co-infected with PEDV (47/94; $50 \%)$, TGEV (1/94; 1.1\%), or PRoV (1/94; 1.1\%). Infection with only PEDV and TGEV was documented in $29 / 314$ (9.2\%) and 5/314 (1.6\%) samples, respectively. The only case of PRoV was a co-infection with PKV. We did not observe

Table 1. Primer pairs used to detect porcine diarrhea viruses in stool and intestinal samples

\begin{tabular}{|c|c|c|c|c|}
\hline Virus & Primer sequence & Product length & Target gene & Reference strain \\
\hline PKV & F:5'-TGGATTACAAGTGTTTT-3' & $217 \mathrm{bp}$ & $3 \mathrm{D}$ & S-1-HUN \\
\hline PEDV & $\begin{array}{l}\text { R:5'-ATGTTGTTAATGATGGT-3' } \\
\text { F:5'-GGACACATTCTTGGTGGT-3' } \\
\text { R:5'-GTTTAGACTAAATGAAG-3' }\end{array}$ & 370 bp & $\mathrm{M}$ & $\begin{array}{c}\text { CV777 } \\
(\mathrm{AF} 353511)\end{array}$ \\
\hline TGEV & $\begin{array}{l}\text { F:5'-GATTTGATTTGGCAATGC-3' } \\
\text { R:5'-AACAATCACTAGATCCAG-3' }\end{array}$ & $102 \mathrm{bp}$ & $7 \mathrm{~b}$ & $\begin{array}{c}\text { WH-1 } \\
\text { (HQ462571) }\end{array}$ \\
\hline PRoV & $\begin{array}{l}\text { F:5'-TGGTATTGAATATACCAC-3' } \\
\text { R:5'-CTGTTGGCCACCCTTTAG-3' }\end{array}$ & 802 bp & VP4 & $\begin{array}{c}\text { JL94 } \\
(\text { AY523636) }\end{array}$ \\
\hline
\end{tabular}

$\mathrm{PKV}=$ porcine kobuvirus; $\mathrm{PEDV}=$ porcine epidemic diarrhea virus; $\mathrm{TGEV}=$ transmissible gastroenteritis virus; $\mathrm{PRoV}=$ porcine rotavirus. 
Table 2. Isolated strains of PEDV and PKV used for sequence alignment and phylogenetic analysis

\begin{tabular}{|c|c|c|c|c|c|}
\hline \multicolumn{3}{|c|}{ PEDV } & \multicolumn{3}{|c|}{ PKV } \\
\hline Strain name & GenBank Acc. No. & Place of origin & Strain name & GenBank Acc. No. & Place of origin \\
\hline JSCZ130818 & KM983619 & Jiangsu & SH1402 & KM983629 & Shanghai \\
\hline HNZK1402 & KM983616 & Jiangsu & GuaNan4201307 & KF977012 & Jiangsu \\
\hline GDGZ1312 & KM983611 & Guangdong & RuGao2201303 & KF977025 & Jiangsu \\
\hline JSBH140210 & KM983618 & Jiangsu & SiPing26201311 & KF977030 & Jilin \\
\hline JSYZ131228 & KM983624 & Jiangsu & SiPing17201311 & KF977029 & Jilin \\
\hline GDGZ13121 & KM983612 & Guangdong & SiPing15201311 & KF977027 & Jilin \\
\hline SH14031 & KM983626 & Shanghai & SiPing16201311 & KF977028 & Jilin \\
\hline HNZY1312 & KM983617 & Henan & GuanYun13201301 & KF977016 & Jiangsu \\
\hline JSTZ1303 & KM983622 & Jiangsu & HaiZhou4201301 & KF977019 & Jiangsu \\
\hline JSYC1303 & KM983623 & Jiangsu & HN13121 & KM983628 & Henan \\
\hline SH1403 & KM983625 & Shanghai & GuanYun27201301 & KF977018 & Jiangsu \\
\hline HNXC1403 & KM983615 & Henan & HN1312 & KM983627 & Henan \\
\hline HNLB1402 & KM983613 & Henan & Haizhou201301 & KF317208 & Jiangsu \\
\hline HNWH1403 & KM983614 & Henan & GuangZ201312 & KJ556981 & Jiangsu \\
\hline JSDF131228 & KM983620 & Jiangsu & ShuY201312 & KJ556982 & Jiangsu \\
\hline JSNT131122 & KM983621 & Jiangsu & & & \\
\hline
\end{tabular}

$\mathrm{PEDV}=$ porcine epidemic diarrhea virus; $\mathrm{PKV}=$ porcine kobuvirus.

Table 3. Reference strains used for phylogenetic comparisons

\begin{tabular}{|c|c|c|c|c|c|}
\hline \multicolumn{3}{|c|}{ PEDV } & \multicolumn{3}{|c|}{ Kobuvirus } \\
\hline Strain name & GenBank Acc. No. & Place of origin & Strain name & GenBank Acc. No. & Place of origin \\
\hline HNQX & JN400902 & China & RBA24 & HQ877620 & Brazil \\
\hline HNBF & JN400903 & China & WUH1 & JQ692069 & China \\
\hline BJ2010 & JF690778 & China & $\mathrm{XX}$ & KC204684 & China \\
\hline DX & EU031893 & China & WB-1-HUN & JX177612 & Hungary \\
\hline LZC & EF185992 & China & THA/2008 & AB624472 & Japan \\
\hline LJB-03 & AY608890 & China & THA/CMP035 & GQ152100 & Japan \\
\hline USA/Colorado/2013 & KF272920 & USA & K-30-HUN & GQ249161 & Hungary \\
\hline PFF188 & FJ687462 & Korea & S-1-HUN & NC_011829 & Hungary \\
\hline IA 2 & KF468754 & USA & swine/2007/CHN & FJ493623 & China \\
\hline $\mathrm{MN}$ & KF468752 & USA & Y-1-CHI & GU292559 & China \\
\hline M2227 & FJ687456 & Korea & $\mathrm{CH} / \mathrm{HNXX}-4$ & JX401523 & China \\
\hline Chinju99 & DQ845249 & Korea & Japan/2009 & HM014081.1 & Japan \\
\hline KPEDV-9 & AF01588 & Korea & CPF4293 & HQ234909 & Korea \\
\hline $\mathrm{Br} 1 / 87$ & $\mathrm{Z} 24733$ & England & $\mathrm{U}-1$ & AB084788 & Japan \\
\hline CV777 & AF353511 & Belgium & TB3/HUN/2009 & GU245693 & Hungary \\
\hline 08RB03 & FJ196184 & Tailand & Aichi virus 1 & AB010145 & Japan \\
\hline M_NIAH1795_04 & EU542415 & Tailand & US-PC0082 & JN088541 & USA \\
\hline M_NIAH2013_95 & EU581711 & Tailand & M-5/USA/2010 & NC_015936 & USA \\
\hline Iowa18984 & KF804028 & USA & & & \\
\hline JS-2004-2 & AY653205 & China & & & \\
\hline
\end{tabular}

$\mathrm{PEDV}=$ porcine epidemic diarrhea virus.

co-infections with more than two of the viruses, and PKV was always one of the co-infecting viruses. There were no cases of co-infection with any combination of PEDV, TGEV, and PRoV.

\section{Sequence analysis of the PKV $3 D$ gene}

The sequence homology of the 3D gene was assessed for 15 PKV strains collected during the study. Of these, 


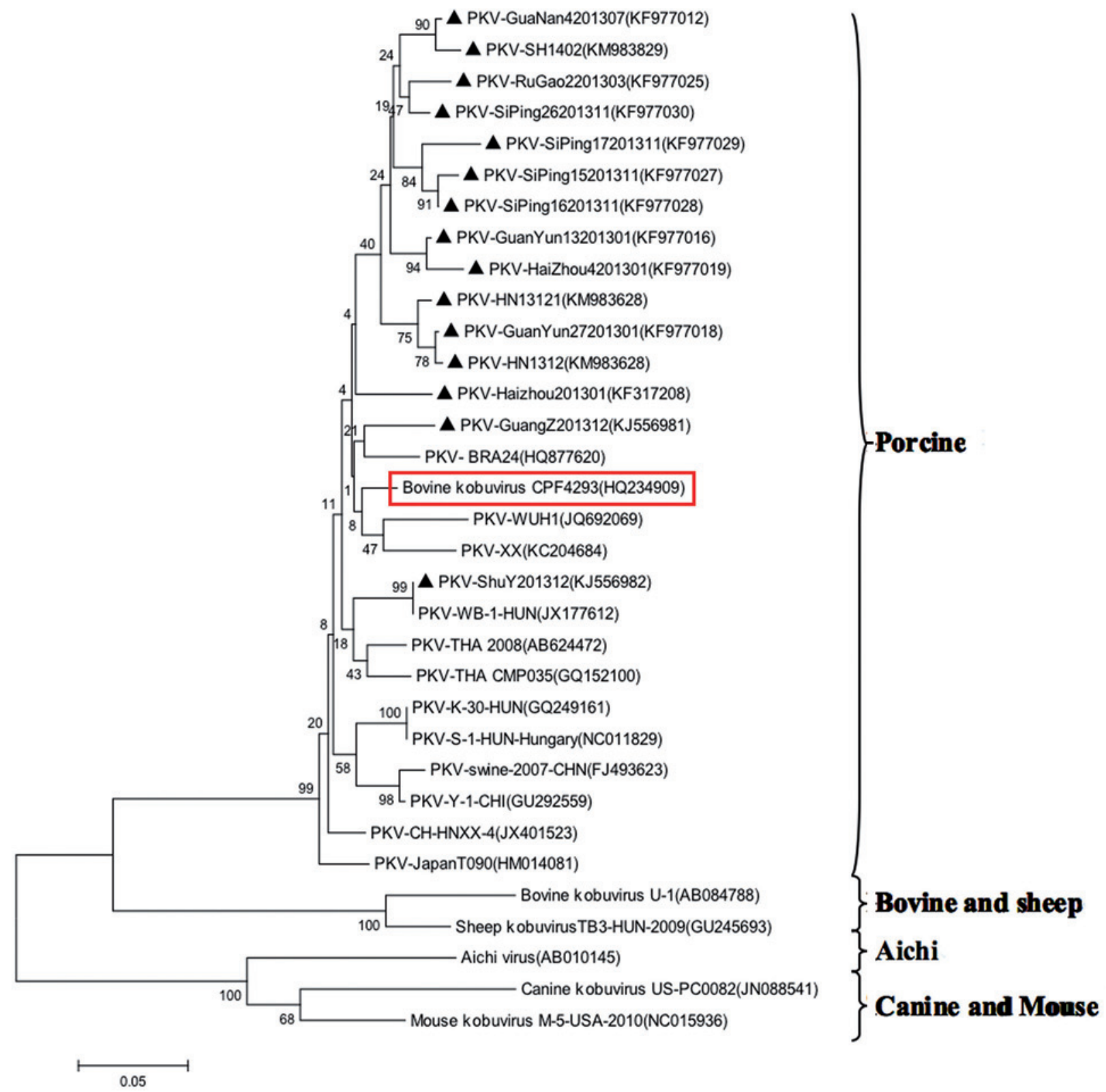

Fig. 1

Phylogenetic analysis by the neighbor-joining method based on partial nucleotide sequences of PKV 3D region (183 bp without primers) Sequence of $15 \mathrm{PKV}$ strains collected is indicated by the filled triangle.

the amplicons demonstrated $86.8-100 \%$ identity on the nucleotide level and $69.4-100 \%$ identity on the amino acid level. The nucleotide and deduced amino acid sequence identity of the PKV strains were then compared to reference strains (Y-1-CHI and S-1-HUN) and other kobuviruses. Compared to Y-1-CHI, the nucleotide identity of the collected PKV strains was $90.3-95.9 \%$ and the deduced amino acid identity was 66.7-87.5\%; compared to S-1-HUN the identities were $88.0-94.9 \%$ for nucle- otide and $73.6-88.9 \%$ for amino acid sequences. Finally compared to other kobuviruses, the nucleotide sequence identity was $69.8-78.8 \%$ and the deduced amino acid identity was $27.8-56.9 \%$. In a phylogenetic analysis, 15 strains collected from different geographical locations in China clustered together with the reference PKV strains, while kobuviruses from other species formed two clusters. Interestingly, a bovine kobuvirus clustered with PKV (Fig. 1). 


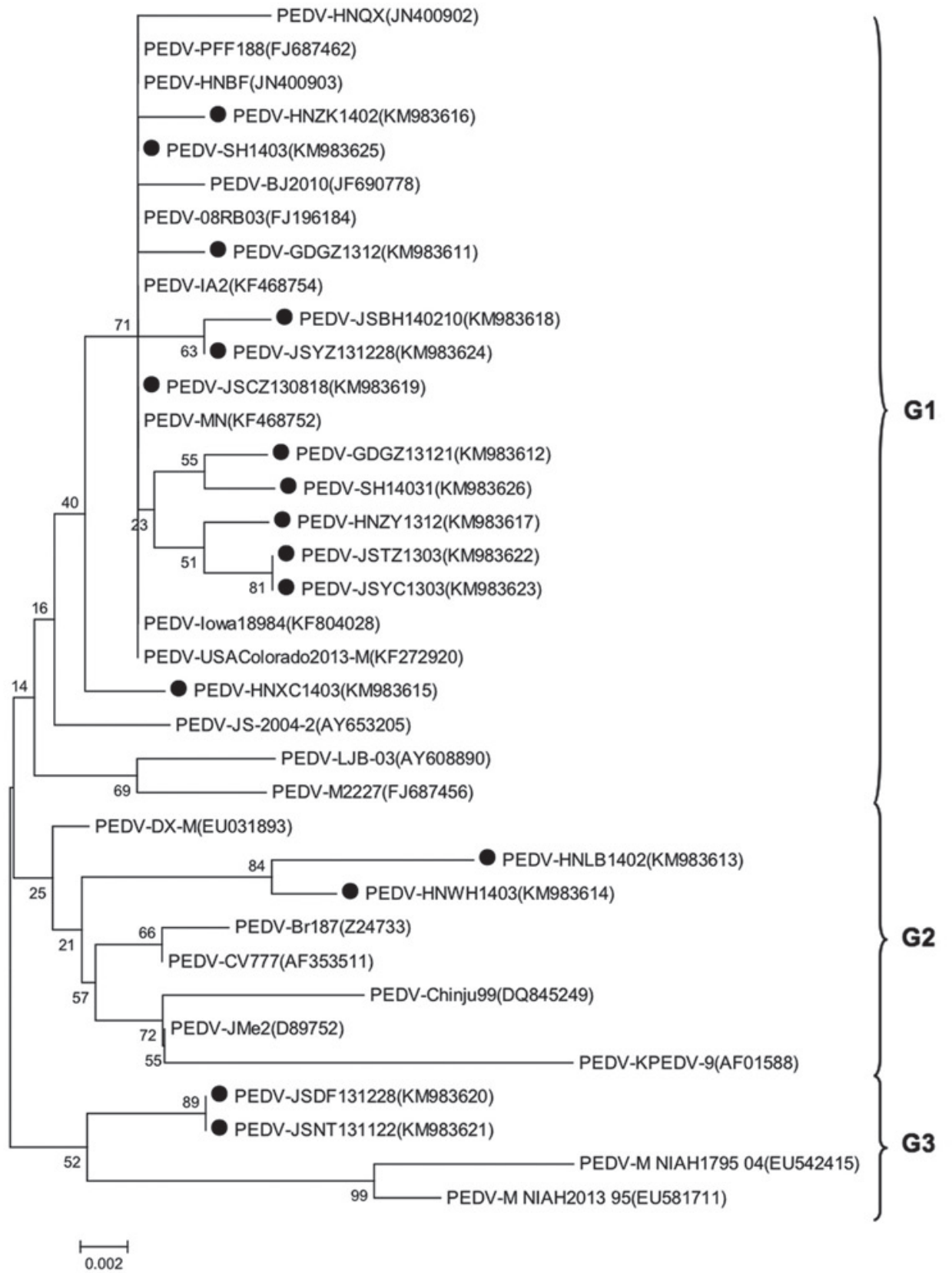

Fig. 2

Phylogenetic analysis by the neighbor-joining method based on the partial nucleotide sequences of the PEDV M region ( 335 bp without primers) The sequences of the 16 PEDV collected strains are indicated by the filled circle.

Sequence analysis of the PEDV M gene

The sequence homology of the PEDV strains collected $(\mathrm{n}=16)$ was based on the partial $\mathrm{M}$ gene sequence. The 16 amplicons showed $96.8-100 \%$ identity at the nucleotide level and $95.9-100 \%$ identity at the amino acid level. The nucleotide and deduced amino acid sequences of the col- lected PEDV strains were then compared to reference strains (CV777 and US) and to other regional PEDV strains. Regarding the reference strains, the nucleotide sequence identity compared to CV777 was $98.1-98.9 \%$ and $97.6-100 \%$ identity at amino acid level compared to US strains. The deduced amino acid identities of the isolates compared to CV777 and US strains were $97.5-99.2 \%$ and $96.7-100 \%$, respectively. 
Compared to other regional PEDV strains, the nucleotide sequence identity was $93.5-100 \%$ and the deduced amino acid sequence identity was $95.9-100 \%$. Phylogenetic analysis based on the partial $\mathrm{M}$ gene sequence resulted in three clusters. The G1 cluster contained 12 PEDV strains collected in China and some reference strains, from the US, Korea, Thailand, and other Chinese regions. Two Henan strains clustered with CV777 in G2 cluster. The G3 cluster contained two Jiangsu strains and two strains from Thailand (Fig. 2).

\section{Discussion}

Porcine diarrhea can be caused by PEDV, TGEV, and PRoV, but PEDV was the pathogen responsible for a porcine diarrhea epidemic in China in 2010 (Chen et al., 2012, 2013b; Gao et al., 2013; Ge et al., 2013; Li et al., 2012; Sun et al., 2012), that resulted in major economic losses in the swine industry. The prevalence of PKV ranges from 19.3-99.0\% in different countries (Wang et al., 2011), and it can infect pigs of all ages and varieties. PKV has also been linked to porcine diarrhea although its pathogenesis remains unclear (Barry et al., 2011; Chen et al., 2013a). Based on the rates of infection documented in this study, PEDV (24.2\%) is still the primary cause of porcine diarrhea in the past two years. The infection rate for PKV is also very high (29.9\%), and of the PKV infected samples, 52.1\% were co-infections with one other virus (PEDV, TGEV, or PRoV), suggesting a high prevalence of co-infection in the sampled regions. Interestingly, co-infections with PEDV and PKV accounted for $61.8 \%$ (47/76) of the PEDV positive samples, which is much higher than the frequency of infection with PEDV alone. Thus, PKV may only be pathogenic as a secondary infection following infection by PEDV or another pathogen (Yang et al., 2014). The high prevalence of co-infection, particularly $\mathrm{PKV}$ and PEDV, is a cause for concern and should be seriously considered.

Based on the genetic analyses using the PKV 3D gene, the 15 amplicon sequences had high homology with reference PKV strains at the nucleotide level, but low homology with other reference kobuvirus. This suggests that the PKV 3D gene is highly conserved. Phylogenetic analysis showed that there was no regional difference in PKV strains as the collected strains were distributed in different branches. Although one of the strains (GuangZ201312) clustered with a strain from Brazil (BRA24) and a second strain (ShuY201312) clustered with a strain from Hungary (WB-1-HUN). These findings indicate that the PKVs circulating in China are genetically diverse. The presence of a bovine kobuvirus in the cluster with PKV suggests that there may be interspecies transmission of kobuviruses (Reuter et al., 2010).

The PEDV M gene was also highly conserved based on genetic analyses. All 16 amplicon sequences had high homol- ogy with reference PEDV strains from GenBank at both the nucleotide and amino acid levels. PEDV clustered into three groups based on the phylogenetic analysis of the amino acid sequences of the M protein. Recently isolated Chinese PEDV strains tended to vary. Most of the strains in this study belonged to the G1 group. There is likely environmental or immunological pressure on PEDV that is keeping the M gene of the Chinese isolates since 2003 tightly clustered, but distinct from the CV777 vaccine strain. American-like isolates were also in this branch, and the PEDV strains causing outbreaks in America recently may be related to the PEDV strain in China (Tian et al., 2014). Two Henan strains belong to the branch which includes CV777, suggesting that CV777 may be their ancestor. Most notably, two Jiangsu strains clustered with two strains from Thailand, which is quite rare. Therefore, a new genotype may be transmitted to China from other places, and could explain why herds immunized with CV777 continue to experience PEDV outbreaks in China. Thus, additional PEDV vaccines based on different genotypes should be developed to prevent and control this disease.

\section{Conclusions}

The rate of infection with PKV and PEDV were very high in different parts of China. The PKV isolates were genetically diverse, while the PEDV isolates were more heterogeneous. Co-infections with PKV and PEDV were very common on pig farms, suggesting that controlling piglet diarrhea might be more complex than previously thought. A better understanding of viruses that cause diarrhea in piglets will aid in better preventing and controlling epidemics of viral piglet diarrhea.

Acknowledgements. This work was supported by the Qing Lan Project, Program for Changjiang Scholars, Innovative Research Team in University (PCSIRT), and the Project Funded by the Priority Academic Program Development of Jiangsu Higher Education Institutions (PAPD).

\section{References}

Barry AF, Ribeiro J, Alfieri AF, van der Poel WH, Alfieri AA (2011): First detection of kobuvirus in farm animals in Brazil and the Netherlands. Infect. Genet. Evol. 11, 1811-1814. http://dx.doi.org/10.1016/j.meegid.2011.06.020

Cao W, Zheng H, Zhang K, Jin Y, Lv L, Yang F, Liu X (2012): Complete genome sequence of the porcine kobuvirus variant CH/HNXX-4/2012. J. Virol. 86, 11947. http://dx.doi. org/10.1128/JVI.02137-12

Chen X, Yang J, Yu F, Ge J, Lin T, Song T (2012): Molecular characterization and phylogenetic analysis of porcine epidemic diarrhea virus (PEDV) samples from field cases 
in Fujian, China. Virus Genes 45, 499-507. http://dx.doi. org/10.1007/s11262-012-0794-X

Chen L, Zhu L, Zhou YC, Xu ZW, Guo WZ, and Yang WY (2013a): Molecular and phylogenetic analysis of the porcine kobuvirus VP1 region using infected pigs from Sichuan Province, China. Virol. J. 10, 281. http://dx.doi. org/10.1186/1743-422X-10-281

Chen X, Zeng L, Yang J, Yu F, Ge J, Guo Q, Gao X, Song T (2013b): Sequence heterogeneity of the ORF3 gene of porcine epidemic diarrhea viruses field samples in Fujian, China, 2010-2012. Viruses 5, 2375-2383. http://dx.doi. org/10.3390/v5102375

Coussement W, Ducatelle R, Debouck P, Hoorens J (1982): Pathology of experimental CV777 coronavirus enteritis in piglets. I. Histological and histochemical study. Vet. Pathol. 19, 46-56. http://dx.doi.org/10.1177/030098588201900108

Gao Y, Kou Q, Ge X, Zhou L, Guo X, Yang H (2013): Phylogenetic analysis of porcine epidemic diarrhea virus field strains prevailing recently in China. Arch Virol. 158, 711-715. http://dx.doi.org/10.1007/s00705-012-1541-2

Ge FF, Yang DQ, Ju HB, Wang J, Liu J, Liu PH, Zhou JP (2013): Epidemiological survey of porcine epidemic diarrhea virus in swine farms in Shanghai, China. Arch Virol. 158, 22272231. http://dx.doi.org/10.1007/s00705-013-1722-7

Khamrin P, Maneekarn N, Hidaka S, Kishikawa S, Ushijima K, Okitsu S, Ushijima H (2010): Molecular detection of kobuvirus sequences in stool samples collected from healthy pigs in Japan. Infect. Genet. Evol. 10, 950-954.

http://dx.doi.org/10.1016/j.meegid.2010.06.001

Laude H, Rasschaert D, Delmas B, Godet M, Gelfi J, Charley B (1990): Molecular biology of transmissible gastroenteritis virus. Vet. Microbiol. 23, 147-154. http://dx.doi. org/10.1016/0378-1135(90)90144-K

Li ZL, Zhu L, Ma JY, Zhou QF, Song YH, Sun BL, Chen RA, Xie QM, Bee YZ (2012): Molecular characterization and phylogenetic analysis of porcine epidemic diarrhea virus (PEDV) field strains in south China. Virus Genes 45, 181-185. http://dx.doi.org/10.1007/s11262-012-0735-8

Park SJ, Lim YS, Hwang S, Heo NY, Lee HC, Suh DJ, Yu E, Lee SG (2010): Emergency adult-to-adult living-donor liver transplantation for acute liver failure in a hepatitis B virus endemic area. Hepatology 51, 903-911. http://dx.doi. org/10.1002/hep.23369

Prabha S, Verghese S (2009): Detection of porcine rotavirus from tissue and faecal specimens. Indian. J. Med. Microbiol. 27, 149-152. http://dx.doi.org/10.4103/0255$\underline{0857.49430}$
Reuter G, Boldizsar A, Kiss I, Pankovics P (2008): Candidate new species of Kobuvirus in porcine hosts. Emerg Infect Dis. 14, 1968-1970. http://dx.doi.org/10.3201/ eid1412.080797

Reuter G, Boros A, Pankovics P, Egyed L (2010): Kobuvirus in domestic sheep, Hungary. Emerg. Infect. Dis. 16, 869-870. http://dx.doi.org/10.3201/eid1605.091934

Shi D, Zhang S, Chen J, Shi H, Zhang X, Feng L (2013): Molecular characterization of a porcine kobuvirus variant strain in China. Arch. Virol. 158, 2379-2383. http://dx.doi. org/10.1007/s00705-013-1736-1

Sisay Z, Wang Q, Oka T, Saif L (2013): Prevalence and molecular characterization of porcine enteric caliciviruses and first detection of porcine kobuviruses in US swine. Arch Virol. 158, 1583-1588. http://dx.doi.org/10.1007/s00705-013 $\underline{-1619-5}$

Sun RQ, Cai RJ, Chen YQ, Liang PS, Chen DK, Song CX (2012): Outbreak of porcine epidemic diarrhea in suckling piglets, China. Emerg. Infect. Dis. 18, 161-163. http://dx.doi. org/10.3201/eid1801.111259

Tamura K, Peterson D, Peterson N, Stecher G, Nei M, Kumar S (2011): MEGA5: molecular evolutionary genetics analysis using maximum likelihood, evolutionary distance, and maximum parsimony methods. Mol. Biol. Evol. 28, 2731-2739. http://dx.doi.org/10.1093/molbev/msr121

Tian PF, Jin YL, Xing G, Qv LL, Huang YW, Zhou JY (2014): Evidence of recombinant strains of porcine epidemic diarrhea virus, United States, 2013. Emerg. Infect. Dis. 20, 1735-1738. http://dx.doi.org/10.3201/eid2010.140338

Wang C, Lan D, Hua X (2011): Porcine kobuvirus from pig stool specimens in Shanghai, China. Virus Genes. 43, 350-352. http://dx.doi.org/10.1007/s11262-011-0643-3

Yang Z, Jin W, Zhao Z, Lin W, Zhang D, Yu E, Qin A, Yang H (2014): Genetic characterization of porcine kobuvirus and detection of coinfecting pathogens in diarrheic pigs in Jiangsu Province, China. Arch Virol. 159, 3407-3412. http://dx.doi.org/10.1007/s00705-014-2204-2

Yu JM, Jin M, Zhang Q, Li HY, Li DD, Xu ZQ, Li JS, Cui SX, Yang SH, Liu N, Duan ZJ (2009): Candidate porcine Kobuvirus, China. Emerg. Infect. Dis. 15, 823-825. http://dx.doi. org/10.3201/eid1505.081518

Zhao J, Shi BJ, Huang XG, Peng MY, Zhang XM, He DN, Pang R, Zhou B, Chen PY (2013): A multiplex RT-PCR assay for rapid and differential diagnosis of four porcine diarrhea associated viruses in field samples from pig farms in East China from 2010 to 2012. J. Virol. Methods 194, 107-112. http://dx.doi.org/10.1016/j.jviromet.2013.08.008 